\title{
SM Otolaryngology Diagnosis and Surgical Treatment of Mucosal Contact Point Headache: Mucosal Contact Point Headache May Not be Accurately Diagnosed before Surgery
}

Jia Wang MS, Jin-shu Yin MS* and Hong Peng BS

Department of Otolaryngology Head and Neck Surgery, Beijing Millennium Monument Hospital affiliated with Capital Medical University, PR China

\section{Article Information}

Received date: Dec 13, 2016

Accepted date: Jan 10, 2017

Published date: Jan 18, 2017

\section{${ }^{*}$ Corresponding author}

Jin-shu Yin MS, Department of Otolaryngology Head and Neck Surgery, Beijing Millennium Monument Hospital, Tieyi Road 10\#, Haidian district, Beijing, China, Tel: 086-15301378578;

Email: yinjinshu@aliyun.com

Distributed under Creative Commons CC-BY 4.0

Keywords Contact point; CT scan;

Deviation; Headache; Intranasal; Nasal septum

Abbreviations CT: Computed Tomography; ICHD: International Classification of Headache Disorders; VAS: Visual Analogue Scale

Article DOI 10.36876/smotol.1003

\section{OPEN ACCESS}

\section{ISSN: 2574-2418}

\section{Abstract}

Objective: We aimed to investigate the differences in incidence of nasal anatomic abnormalities between the patients with and without headache and the outcome of surgical treatment for the headache patients with mucosal contact point.

Background: "Mucosal contact point headache" has been defined by Headache Classification Committee of the International Headache Society. Whether the type of nasal anatomic abnormalities associates with incidence of headache and whether surgical treatment is necessary remain undefined.

Study Design: Observational study.

Methods: We recruited 107 subjects without headache and 78 subjects with refractory headache for more than 2 years. Subjects underwent high-resolution sinus CT scans and the incidence of nasal anatomic abnormalities was calculated in both groups and results were compared. An additional 25 patients underwent surgical treatment.

Results: Mucosal contact points were observed in $85.9 \%$ of patients with refractory headache and also in $80.4 \%$ of subjects without headache. The most common mucosal contact point among headache group patients was between deviated nasal septum and lateral nasal wall (55.1\%) and the incidence of this type of contact point was significantly different between groups $(P<0.05)$. An additional 25 headache patients with mucosal contact point were corrected via endoscopic surgery. Average pain scale scores decreased significantly between pre- and post-operative measurements $(P<0.001)$. Only $44 \%$ of patients had recovered from headache 7 days postoperatively.

Conclusion: Some patients with recurrent headache and mucosal contact point may not meet the diagnostic criteria of mucosal contact point headache since pain was not resolved within 7 days after surgical treatment.

\section{Introduction}

Headache may result when pressure exerted on the nasal mucosa in the contact areas by anatomical variations, polyps, or mucosal swelling initiates the release of substance $\mathrm{P}$, which triggers pain impulses in afferent $C$ fibers [1].

With the rapid development of medical imaging and endoscopic techniques, abnormal nasal anatomy has been found to have multiple manifestations, including agger nasi cells, Haller cells, pneumatization of the middle turbinate, middle turbinate abnormal curve, uncinate process abnormalities (hyperplasia, deviation, and pneumatization), nasal septum deviation, ethmoid bulla and maxillary sinus hypoplasia [2]. Contact points refer to intranasal contact between opposing mucosal surfaces. In 1980, in their experience with middle turbinectomy, Morgenstein and Krieger [3] discovered that deformity of turbinate anatomy could cause headache and were the first investigators to propose the concept of contact point headache.

The concept of contact point headache has been widely cited by the Society of OtolaryngologyHead and Neck Surgery and has been applied in clinical practice [4]. Mucosal contact headache was recently added as secondary headache disorder in the second edition of the International Classification of Headache Disorders (ICHD-2), 4 where it is described as being characterized by intermittent pain localized in the periorbital and medial canthal or temporozygomatic regions, associated with evidence of mucosal contact points by nasal endoscopy or Computed Tomography (CT) imaging. Research has shown that different anatomical variations, especially septal spurs and abnormal turbinates, can be the cause of intranasal mucosal contacts [5] Pneumatized superior turbinate process or Haller's cell has been reported to cause migraine-type headaches [2]. CT 
scans of the nose and paranasal sinuses of a child with recurrent attacks of unilateral headache showed a spur in contact with the left inferior turbinate [6] however, whether the type of nasal anatomic abnormalities associates with incidence of headache remain undefined. Surgical treatment has been recommended for the patients with refractory primary headaches associated with mucosal contact point $[7,8]$. However, surgery for removal of nasal contact points in patients with facial pain or headache may not be unnecessary $[9,10]$.

The purpose of this study was: (1) to investigate the differences in incidence of mucosal contact points within the nasal cavity in subjects with and without headache; (2) to investigate whether surgical treatment is necessary for the patients who fulfill most part of the diagnostic criteria for mucosal contact point headache.

\section{Materials and Methods}

\section{Participants}

This study was approved by the Institutional Review Board of Beijing Millennium Monument Hospital affiliated with Capital Medical University. In this observational study, 185 healthy volunteers who had undergone physical examination and were patients at the Ear, Nose and Throat clinic or the Department of Neurology between September 2005 and July 2010 were recruited by convenience sampling. Of these, a headache group included 78 patients with a single symptom of refractory headache for more than 2 years. A nonheadache group included 107 patients who had visited the Ear, Nose and Throat clinic but without headache. Patients with nasal-borne diseases, including sinusitis, nasal polyps, nasal cavity and paranasal sinus tumors, and medical conditions that could induce headache (e.g., hypertension) were excluded. All participants provided signed informed consent and underwent sinus high-resolution CT scan.

\section{High-resolution CT (HRCT)}

The Siemens Sensation 16-slice spiral CT was used (Siemens, Erlangen, Germany), and the scan baseline was parallel to the orbitomeatal line. The scanning parameters included collimation: $0.75 \mathrm{~mm}$ $\mathrm{x} 16$, pitch 0.55 . Three-dimensional reconstruction of images was created. Slice thickness was $0.75 \mathrm{~mm}$, slice interval was $0.5 \mathrm{~mm}$, the matrix was $512 \times 512$, the window level was $400 \mathrm{HU}$, and window width was $1800 \mathrm{HU}$, which together ensured the continuity of image information. The same researcher reviewed the images for more than three times at different times (test-retest) to ensure consistency of results of the nasal cavity and sinus anatomical structures. The incidence of various nasal anatomical abnormalities was calculated, and the difference in the incidence of mucous contact points was compared between the headache group and non-headache group.

\section{Diagnostic criteria of mucosal contact point headache}

In our study, the diagnosis of mucosal contact point headache referred to the diagnostic criteria of "Mucosal contact point headache" proposed by Headache Classification Committee of the International Headache Society [4]

A. Intermittent pain localized to the periorbital or medial canthal or temporozygomatic regions fulfilling criteria $\mathrm{C}$ and $\mathrm{D}$.

B. Clinical, nasal endoscopic and/or CT imaging evidence of mucosal contact points without acute sinusitis.
C. Evidence that the pain can be attributed to mucosal contact based on at least one of the following:

1. Pain corresponds to gravitational variations in mucosal congestion as the patient moves between upright and recumbent positions.

2. Abolition of pain within 5 minutes after diagnostic topical application of local anesthesia to the middle turbinate using placebo or other controls.

D. Pain resolves within 7 days, and does not recur, after surgical removal of mucosal contact points.

\section{Intranasal endoscopic surgery}

During the same study period, a total of 25 additional patients (other than 185 healthy volunteers) from the Ear, Nose and Throat clinic who had refractory headache symptoms and were diagnosed as nasal mucosal contact by high-resolution CT without acute sinusitis underwent intranasal endoscopic correction of nasal anatomic abnormalities. Those patients were originally diagnosed as having migraine (9 patients), tension headache (3 patients) and vascular headache (1 patient). However, before surgery, those patients also fulfilled the diagnostic criteria A to $\mathrm{C}$ of "Mucosal contact point headache," including abolition of pain with local anesthesia within 5 min after diagnostic topical application of local anesthesia using placebo or other controls [4]. Among these patients, 17 were males and 8 were females. Mean age was 31.9 years. The course of disease ranged from 2 to 20 years. All participants provided signed informed consent and underwent both nasal endoscopy and sinus highresolution CT scan examination to confirm the diagnosis. Pre- and post-operative CT scan images of the sinuses were collected and the pain scores (Visual Analogue Scale, VAS) were recorded pre- and post-operatively as previously described [11].

\section{Surgical procedure}

All 25 patients underwent functional nasal endoscopic surgery under general anesthesia. The basic principle of surgical treatment was that it should be minimally invasive, that is, the objective was to remove the mucosal contact point while preserving normal anatomical structures of the nasal cavity. Septoplasty was performed for patients with nasal septum deviation, and resection of the spinous process was done for patients with contact between the spinous process of the nasal septem and the lateral wall of the nasal cavity. For patients with nasal septum deviation combined with abnormal anatomy of the middle turbinate, septoplasty was performed first and then turbinoplasty was carried out. Resection of the superior turbinate or ethmoidectomy was performed for patients with contact between the nasal septum and the olfactory cleft. External relocation and radiofrequency ablation of the inferior turbinate were carried out for patients with inferior turbinate hypertrophy.

\section{Statistical analysis}

Comparability between the patients with and without headache was tested using Chi-square test for categorical variables and data were represented by numbers (n) and percentage (\%). Paired t test was used to determine differences in Visual Analogue Scale (VAS) pre- and post-operatively and data were represented as mean \pm Standard Deviation (SD). All statistical assessments are two sided and 
Table 1: Incidence of nasal anatomic abnormality determined by sinus high-resolution CT.

\begin{tabular}{|c|c|c|c|}
\hline Nasal anatomic abnormality & Number of cases showing abnormality & Number of sites showing abnormality ${ }^{b}$ & Incidence ${ }^{c}$ \\
\hline Deviation of nasal septum (NS) & 76 & 76 & $41.1 \%$ \\
\hline Pneumatization of middle turbinate & 60 & 83 & $32.4 \%$ \\
\hline Pneumatization of superior turbinate & 40 & 71 & $21.6 \%$ \\
\hline Abnormal curve of middle turbinate (MT) & 37 & 65 & $20.0 \%$ \\
\hline Pneumatization of nasal septum & 30 & 30 & $16.2 \%$ \\
\hline Hypertrophy of inferior turbinate & 17 & 30 & $9.2 \%$ \\
\hline Haller's cell & 10 & 12 & $5.4 \%$ \\
\hline Pneumatization of uncinate process (UP) & 7 & 10 & $3.8 \%$ \\
\hline Hypertrophy of middle turbinate & 5 & 8 & $2.7 \%$ \\
\hline Node of nasal septum & 3 & 3 & $1.6 \%$ \\
\hline Hypertrophy of uncinate process & 3 & 5 & $1.6 \%$ \\
\hline Abnormal curve of superior turbinate & 2 & 4 & $1.08 \%$ \\
\hline Nasal septum dislocation & 1 & 1 & $0.5 \%$ \\
\hline Medially bent uncinate process & 1 & 2 & $0.5 \%$ \\
\hline Hypertrophy of agger nasi & 1 & 2 & $0.5 \%$ \\
\hline
\end{tabular}

${ }^{a}$ Number of patients showing anatomical abnormalities. The same patient showing more than one abnormality was considered as two or more cases.

${ }^{b}$ Number of sites showing anatomical abnormalities. Some of the cases showing bilateral abnormality were considered as two sites.

${ }^{\mathrm{C}}$ Incidence was the number of cases divided by total number of patients.

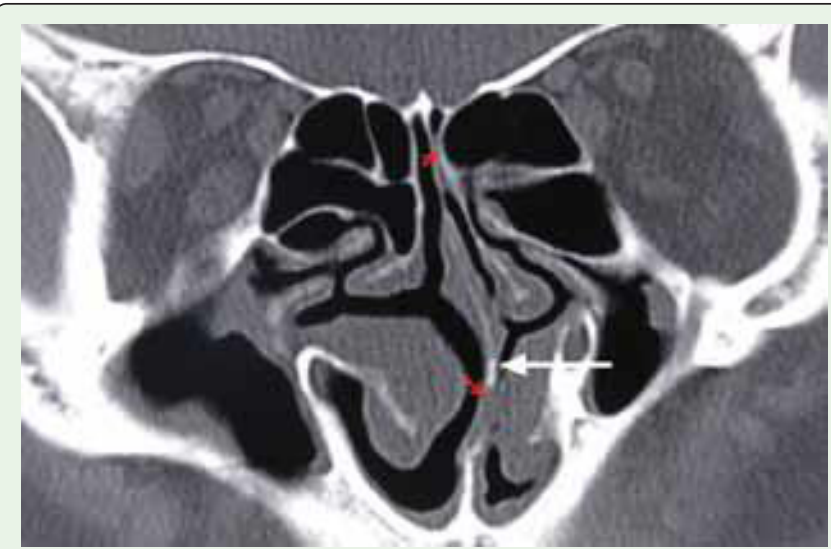

Figure 1: Deviation of nasal septum in contact with left inferior turbinate and left olfactory fissure. evaluated at the 0.05 level of significance. All statistical analyses were performed using SPSS 15.0 statistics software (SPSS Inc, Chicago, IL, USA).

\section{Results}

The incidence of nasal anatomic abnormality among all subjects is shown in Table 1 . The most common nasal anatomic abnormality was the nasal septum deviation (41.1\%) (Figure 1), followed by pneumatization of middle turbinate (32.4\%) (Figure 2). Some patients showed more than one abnormality and were included as two or more cases in Table 1. For example, one patient had hypertrophy of bilateral inferior turbinate in contact with the nasal septum, and pneumatization of the bilateral superior turbinate in contact with the nasal septum (Figure 3). Anatomical abnormalities were either unilateral (Figure 4) or bilateral (Figure 2).

Table 2 represents comparisons of abnormal nasal mucosa contact points between the headache and non- headache groups, including 67 patients in the headache group who had abnormal nasal

Table 2: Comparisons of abnormal nasal mucosa contact between patients with headache and without headache.

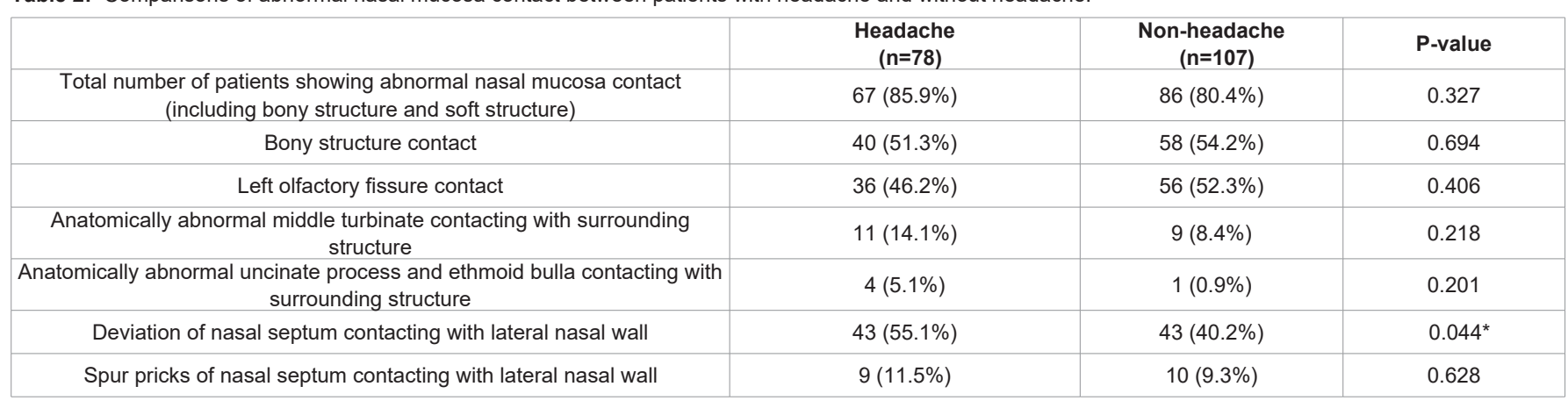

"Indicates a significant difference between the 2 group, $\mathrm{P}<0.05$.

Citation: Wang MSJ, Yin MSJ and Peng BSH. Diagnosis and Surgical Treatment of Mucosal Contact Point Headache: Mucosal Contact Point Headache May Not be Accurately Diagnosed before Surgery. SM Otolaryngol. 2017; 1(1): 1003.

https://dx.doi.org/10.36876/smotol.1003 


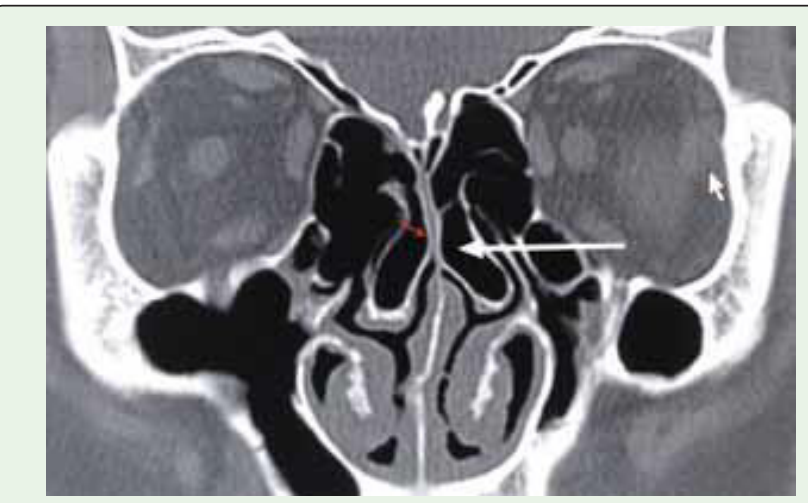

Figure 2: Pneumatization of bilateral middle turbinate in contact with nasal septum.

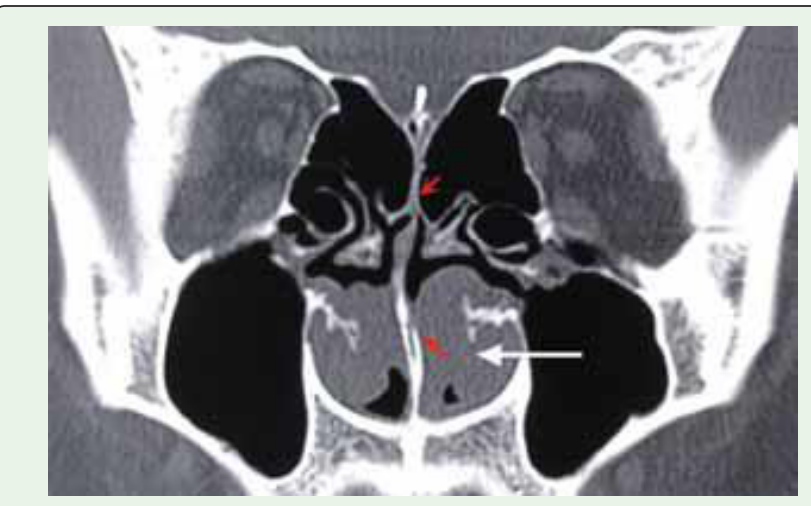

Figure 3: Hypertrophy of bilateral inferior turbinate in contact with nasal septum; pneumatization of bilateral superior turbinate in contact with nasal septum.

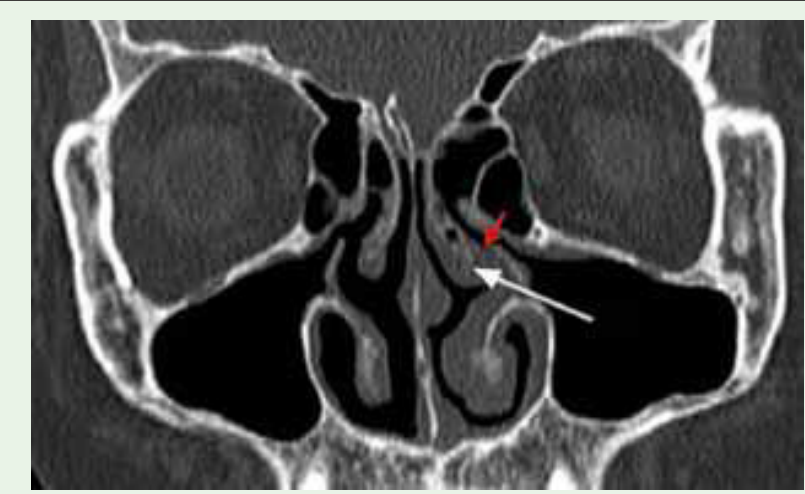

Figure 4: Hypertrophy of left middle turbinate in contact with left uncinate process (UP).

mucosal contact, both bony structure and soft structure contact; and 11 patients (14.1\%) who showed abnormal middle turbinate in contact with surrounding nasal mucosa. A total of 86 (80.4\%) subjects in the non-headache group showed abnormal nasal mucosa contact after CT scan examination. Significant differences were found in the incidence (\%) of nasal septal deviation with a contact point on the lateral nasal wall between patients with and without headache (55.1\% vs. $40.2 \%, \mathrm{P}=0.044$ ) (Table 2). No significant differences were shown between headache and non-headache groups in the other abnormalities.

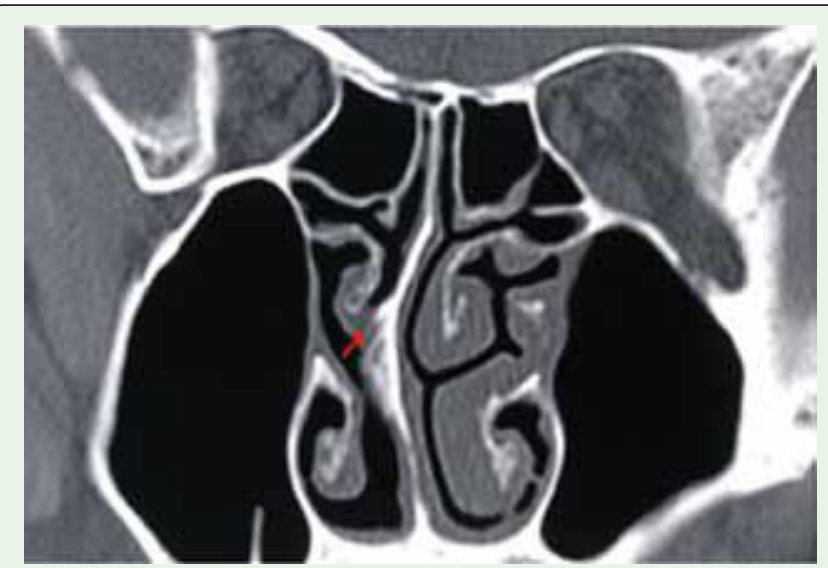

Figure 5A: CT image showing correction of intranasal anatomic abnormalities. Pre-operative spur in contact with right middle turbinate.

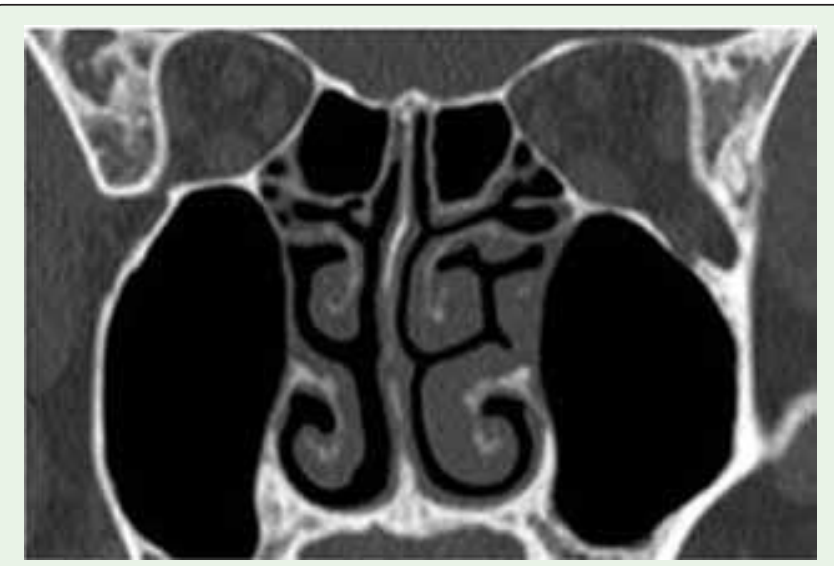

Figure 5B: post-operative elimination of contact point.

Among 25 patients receiving surgical treatment, 10 cases had spur pricks of nasal septum associated middle turbinate and inferior turbinate contact with surrounding structure, 6 cases had deviation of nasal septum associated middle turbinate and inferior turbinate contact. Other abnormalities included olfactory fissure contact (4 cases), pneumatization of middle turbinate (4 cases), and abnormal curve of middle turbinate ( 3 cases) and hypertrophy of turbinate ( 3 cases). The same patient showing more than one abnormality was considered as two or more cases. Figure 5 showed CT image before and after correction of intranasal anatomic abnormalities. After the operation, pain scale scores (VAS) decreased significantly from $60.60 \pm 20.02$ (pre-operation) to $26.20 \pm 27.92$ (post-operation) $(\mathrm{P}<0.001)$ (Figure 6). Eleven patients $(44 \%)$ recovered completely from headache symptoms (VAS score 0 ) 7 days after the surgery and did not recurred after the surgery, suggesting that those patients also fulfilled the diagnostic criteria D of "Mucosal contact point headache" proposed by Headache Classification Committee of the International Headache Society [4]. Eight patients (32\%) showed partial recovery, and 6 patients $(24 \%)$ had the same intensity of pain. Among those 6 patients, 2 patients had complete headache relief (VAS score 0) during the first year after surgery but the headache recurred during the follow-up period (12-18 months post-operative). 


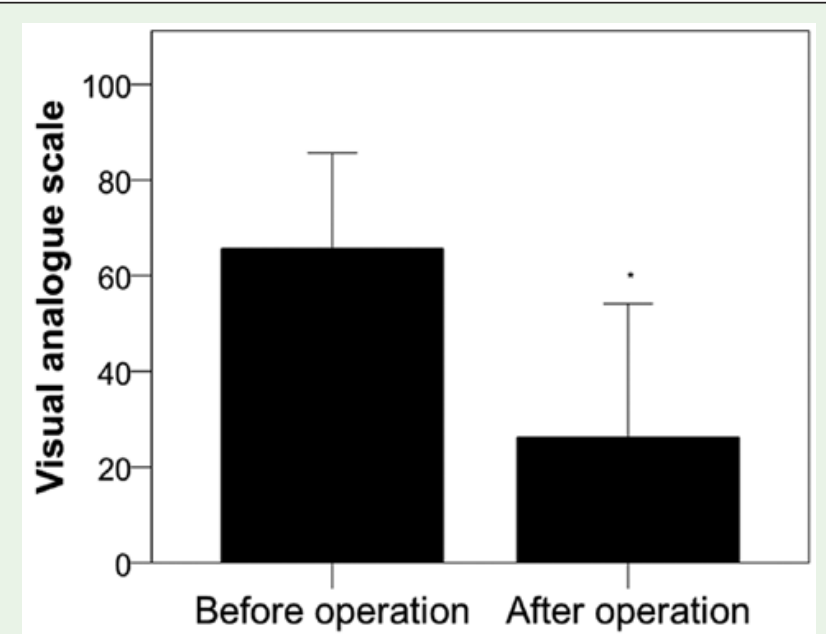

Figure 6: Pain scale results before and after operation.

\section{Discussion}

We investigated whether nasal mucosal contact points caused by nasal anatomic abnormalities could be a cause of refractory headache. We observed mucosal contact points in $85.9 \%$ of patients with refractory headache but contact points were also found in $80.4 \%$ of subjects without headache. The most common nasal anatomical abnormality was nasal septum deviation, and a significant difference was found in the incidence of deviated septum and contact points on the lateral nasal wall between those with headache and those without, suggesting an association between these conditions and headache. However, the incidence of other types of mucosal contact points was not significantly different between the two groups, suggesting that contact points at the spinous process over the septum, in the olfactory cleft area and those caused by anatomic abnormalities of the middle turbinate, uncinate process, and ethmoid bulla might not be associated with headache.

The average post-operative pain scale scores of 25 patients with headache and mucosal contact points decreased significantly after corrective surgery, suggesting that mucosal contact points may be associated with headache. Only eleven patients (44\%) had recovered completely from headache symptoms at 7 days after the surgery and headache had not recurred after the surgery, suggesting that only those patients had fulfilled the diagnostic criteria A to D of "Mucosal contact point headache" proposed by the Headache Classification Committee of the International Headache Society.4 There were still $24 \%(6 / 25)$ of patients who had fulfilled the diagnostic criteria A to C but failed to recover from headache. In addition, we must consider that $80 \%$ of subjects showing abnormal nasal mucosa contact on CT scan examination did not have headache symptoms, and approximately $14 \%$ of subjects with headache did not have abnormal nasal mucosa contact, suggesting that headache had multiple causes. In fact, those 25 patients who had received surgical treatment in our study also suffered from migraine, tension headache and vascular headache. Thus, the etiology and pathology behind those types of headache might be exceptionally diverse. Taken together, our findings suggest that mucosal contact points do not result in refractory headache in most cases, and mucosal contact point headaches may not be accurately diagnosed before surgery.
Contact points are acknowledged as one of the causes of secondary headache or an aggravating factor for primary headache, 4 and are also reported to trigger primary headache (migraine, cluster, tensiontype) [7]. A previous study found that $74 \%$ (73/99) of subjects with various primary headaches had underlying rhinologic abnormalities [12], suggesting that subjects with primary headache might have to be examined by both neurologists and otolaryngologists. However, our study observed $76 \%$ of recovery or partial recovery from headache after the surgery, which was consistent with previous reports. Relief of mucosal contact point headache was reported in $90 \%$ of patients after endonasal surgery, with complete relief in $43 \%$ and improvement in $47 \%$ [2]. In the report of Clerico et al., $79 \%$ of patients with refractory primary headaches responded to endoscopic sinonasal surgery with alleviation of pain severity or headache frequency [7]. Similarly, based on comparisons of pre- and post-operative pain questionnaires, $83 \%$ of patients in another study had recovered from headaches and $8 \%$ had significant relief post-operatively [8]. Removal of contact points in patients with rhinogenic mucosal contact point headache was effective only in carefully selected patients [9]. Surgery for removal of nasal contact points in patients with facial pain is usually unnecessary since prevalence is the same in patients with or without facial pain, indicating that a more central process is responsible [10]. Thus, for some patients with refractory primary headaches, mucosal contact points may not be the only or major cause of the headache, and surgical correction of nasal anatomic abnormalities may not be the proper treatment.

Results of the present study revealed that a greater percentage of patients $(55.1 \%)$ had refractory headache than subjects without headache (40.2\%) had nasal septum deviation and a contact point on the lateral nasal wall. Nasal septum deviation accompanied by nasal ventilation disorders, in which the sinuses may be under negative pressure, could result in headache [13]. Currently there is no direct evidence that substance $\mathrm{P}$ is produced in response to mucosal contact points. However, Blumenthal suggested that mucous contact points promoted release of substance P by local sensory nerve endings, which may act on the nasal mucosa, causing headache [14]. In migraine patients, mucosal contact points may provide additional input, decreasing the threshold for migraine attacks [15]. Thus, a contact point on the lateral nasal wall may stimulate the local sensory nerve or nasal mucosal receptors, which release substance P both centrally and peripherally and can cause headache.

Contact point headache patients may seek medical help at the Department of Neurology, where they may be diagnosed with migraine, cluster headache and tension headache. Although they may undergo long-term pharmacologic treatment, treatment effects may be poor. In the present study, among 78 patients with refractory headache, many had taken pain medicine for more than two years without alleviating their headache symptoms. For patients who fail to respond to conservative treatment, sinus CT scans should be a routine examination for patients with headache, even in the absence of sinus conditions [1]. In addition, Welge-Leussen, et al. [16] suggests that these patients should be checked at the Department of OtolaryngologyHead and Neck Surgery in addition to the Department of Neurology. Careful nasal endoscopy is essential to determine whether mucosal contact points are present. The pathogenesis of headache is complex and diverse and mucosal contact points are not the only cause of refractory headache. 
Based on our results, we suggest that a more strict and scientific standard should be developed for selecting contact point headache surgery cases and long-term follow-up should be carried out to assess the short-, mid- and long-term recovery rates, efficacy rates and recurrence rates of surgical resection of mucosal contact points for treatment of headache. Further studies are necessary to demonstrate the significance and necessity of surgical treatment.

In conclusion, nasal septum deviation with a contact point between the deviated nasal septum and the lateral nasal wall is not necessarily associated with headache. Patients who have fulfilled the diagnostic criteria A to C of "Mucosal contact point headache" proposed by the Headache Classification Committee of the International Headache Society may still fail to recover from headache after corrective surgery.

\section{Acknowledgement}

Thank my mentor Yin Jinshu for giving me guidance in the course of my research, Dr. Peng Hong for surgeries support, thanks all members of our team, there would be no such research without you, thank you.

\section{References}

1. Stammberger $\mathrm{H}$, Wolf $\mathrm{G}$. Headaches and sinus disease: the endoscopic approach. Ann Otol Rhinol Laryngol Suppl. 1988; 134: 3-23.

2. Tosun F, Gerek M, Ozkaptan Y. Nasal surgery for contact point headaches. Headache. 2000; 40: 237-240.

3. Morgenstein $\mathrm{KM}$, Krieger MK. Experiences in middle turbinectomy Laryngoscope. 1980; 90: 1596-1603.

4. Headache Classification Subcommittee of the International Headache Society. The International Classification of Headache Disorders: 2nd edition Cephalalgia. 2004; 24: 9-160.
5. Parsons DS, Batra PS. Functional endoscopic sinus surgical outcomes for contact point headaches. Laryngoscope. 1998; 108: 696-702.

6. Mishra D, Choudhury KK, Gupta A. Headache with autonomic features in a child: cluster headache or contact-point headache? Headache. 2008; 48: 473-475.

7. Clerico DM, Evan K, Montgomery L, Lanza DC, Grabo D. Endoscopic sinonasal surgery in the management of primary headaches. Rhinology. 1997; 35: 98-102.

8. Behin F, Behin B, Behin D, Baredes S. Surgical management of contact point headaches. Headache. 2005; 45: 204-210.

9. Bektas D, Alioglu Z, Akyol N, Ural A, Bahadir O, Caylan R. Surgical outcomes for rhinogenic contact point headaches. Med Princ Pract. 2011; 20: 29-33.

10. Abu-Bakra M, Jones NS. Prevalence of nasal mucosal contact points in patients with facial pain compared with patients without facial pain. J Laryngol Otol. 2001; 115: 629-632.

11. Giacomini PG, Alessandrini M, DePadova A. Septoturbinal surgery in contact point headache syndrome: long-term results. Cranio. 2003; 21: 130-135.

12. Yazici ZM, Cabalar M, Sayin I, Kayhan FT, Gurer E, Yayla V. Rhinologic evaluation in patients with primary headache. J Craniofac Surg. 2010; 21 1688-1691.

13. Behin F, Lipton RB, Bigal M. Migraine and intranasal contact point headache: is there any connection? Curr Pain Headache Rep. 2006; 10: 312-315.

14. Blumenthal HJ. Headaches and sinus disease. Headache. 2001; 41: 883888.

15. Behin F, Behin B, Bigal ME, Lipton RB. Surgical treatment of patients with refractory migraine headaches and intranasal contact points. Cephalalgia. 2005; 25: 439-443.

16. Welge-Luessen A, Hauser R, Schmid N, Kappos L, Probst R. Endonasal surgery for contact point headaches: a 10-year longitudinal study. Laryngoscope. 2003; 113: 2151-2156. 